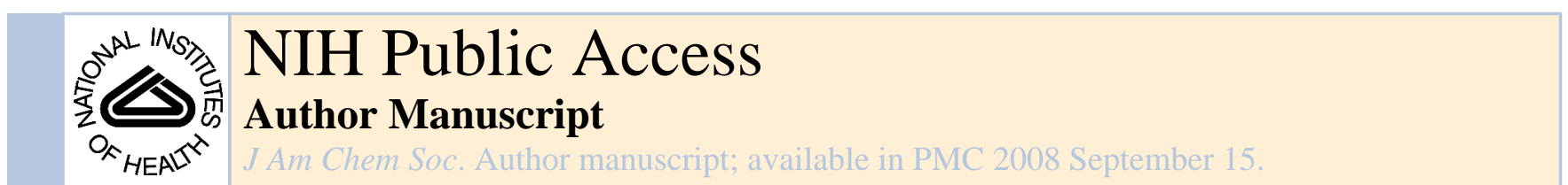

Published in final edited form as:

J Am Chem Soc. 2006 November 29; 128(47): 15050-15051. doi:10.1021/ja0647708.

\title{
Iron-Catalyzed Carbometalation of Propargylic and Homopropargylic Alcohols
}

\author{
Donghui Zhang and Joseph M. Ready ${ }^{\star}$ \\ Department of Biochemistry, The University of Texas Southwestern Medical Center at Dallas, 5323 \\ Harry Hines Boulevard, Dallas, TX 75390-9038
}

Olefins are fundamental building blocks for organic synthesis, and a multitude of methods exist for their preparation. Among various approaches, additions to alkynes offer the promise of convergence and stereoselectivity while leveraging the wealth of literature related to alkyne synthesis. Indeed, carbometalation of terminal alkynes represents a general method and has found widespread use in the synthesis of complex molecules. ${ }^{1}$ In contrast, electronically unbiased internal alkynes react slowly and with poor regioselectivity. However, polar functionality near the alkyne has been found to affect both the rate and regioselectivity of carbometalation reactions (eq 1-2). ${ }^{2}$ For example, addition of Grignard reagents to propargylic alcohols introduces an organic fragment proximal to the alcohol (eq 1). ${ }^{2 \mathrm{~b}, \mathrm{c}}$ The opposite regioselectivity has been observed in reactions of homopropargylic alcohols and ethers with vinyl titanium reagents, ${ }^{2 \mathrm{~d}}$ allyl magnesium bromide, ${ }^{2 \mathrm{e}, \mathrm{f}}$ butyl lithium ${ }^{2 \mathrm{~g}}$ and trimethyl aluminum $^{2 \mathrm{~h}}$ (eq $2, \mathrm{n}=2 ; \mathrm{X}=\mathrm{H}$ or alkyl). Here we report an iron-catalyzed carbomagnesiation of propargylic and homopropargylic alcohols which generates tri- and tetra-substituted olefins with high regio- and stereoselectivity (eq $2 ; \mathrm{n}=1-2 ; \mathrm{X}=\mathrm{H}$ ).

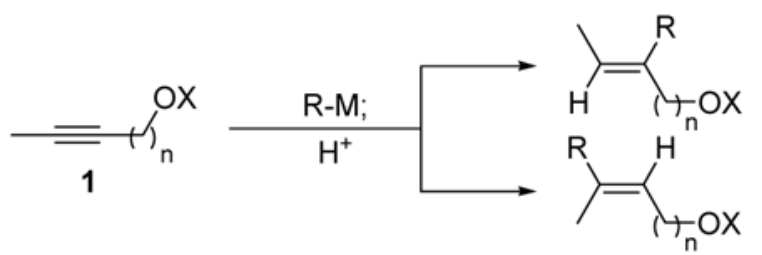

(1), (2)

In the absence of catalysts $\mathrm{CH}_{3} \mathrm{MgBr}$ does not add to the internal propargylic alcohol $\mathbf{1 a}$. Accordingly, we evaluated a variety of first-row transition metal salts for their ability to catalyze methylmagnesiation (Table 1). While $\mathrm{Cu}(\mathrm{I})$ salts did not effect addition, ${ }^{3} \mathrm{Co}(\mathrm{II}), \mathrm{Ni}$ (II) and $\mathrm{Fe}$ (III) displayed promising catalytic activity (entries 2-4). In all cases regioselectivity and stereoselectivity was very high, but the desired trisubstituted olefin (2a) was contaminated with the dimethyl product 3a. The formation of this side product was largely suppressed when bis(diphenylphosphino)ethane (dppe, 1 equiv to $\mathrm{Fe}$ ) was included in the reaction mixture (entry 6). Interestingly, methyllithium proved totally unreactive in the presence or absence of catalyst (entry 7). $2 \mathrm{~g}$

Table 2 presents the generality of the carbometalation. In the presence of $\mathrm{Fe}(\mathrm{acac})_{3}$ or $\mathrm{Fe}$ $(\mathrm{ehx})_{3}(\mathrm{ehx}=2$-ethyl hexanoate) propargylic alcohols react with methyl magnesium bromide to yield trisubstituted allylic alcohols as single regio- and stereoisomers. ${ }^{4}$ Primary and 
secondary propargylic alcohols represent suitable substrates (entries 1-8), the latter reacting with complete conservation of optical purity (entry 7). Common oxygen protecting groups, olefins and tertiary nitrogens appear well-tolerated. Furthermore, primary and secondary homopropargylic alcohols provide the corresponding homoallylic alcohols on treatment with methyl Grignard reagent (entries 9-10,12). In ongoing studies, we have found that the ironcatalyzed carbometalation can be extended to phenylation ${ }^{5}$ (entries 11-12) and ethylation (entries 13-14). In the latter experiments, no diethylation products (analogous to 3) were observed; instead, we obtained small amounts of the product arising from formal cishydrogenation (see below). 6,7

Iron(III) salts are proposed to undergo ligand exchange and reduction with $\mathrm{CH}_{3} \mathrm{MgBr}$ to yield $\mathrm{L}_{\mathrm{n}} \mathrm{Fe}^{\mathrm{II}}\left(\mathrm{CH}_{3}\right)_{2}$ complexes at $0{ }^{\circ} \mathrm{C} .{ }^{8 \mathrm{~b}}$ Lower oxidation states are available upon warming or in the presence of longer chain Grignard reagents. ${ }^{9}$ Accordingly, the oxidation sate of the catalytically active species here remains ambiguous. ${ }^{8 \mathrm{a}}$ Regardless, alkoxide-directed carbometalation likely yields an intermediate (vinyl)Fe species (Scheme 1). In principle, direct coordination to the iron center could occur (4). Alternatively the interaction could be driven by association of iron with magnesium (5). ${ }^{10}$ The (vinyl)Fe(R) species can undergo metathesis with Grignard reagent to provide the carbometalated product and regenerate catalyst, or it can suffer reductive elimination to yield the geminally dialkylated product 3 . $\beta$-Hydride elimination from an $\mathrm{Fe}$ (ethyl) intermediate could give rise to an $\mathrm{Fe}-\mathrm{H}$ species 6. Subsequent hydrometalation could lead to the hydrogenated side products observed in ethylation reactions.

The presumptive vinyl Grignard intermediate $\mathbf{7}$ can be trapped with a variety of electrophiles to yield tetrasubstituted allylic alcohols. For example, deuteration, formylation, allylation and bromination proceeds under the conditions indicated in Scheme 2. Likewise, trapping with benzaldehyde provides the allylic alcohols $\mathbf{8}$ and $\mathbf{9}$ as single olefin isomers. Finally, trapping the vinyl iron or magnesium species with a pendant alkyne yields the cyclic diene $\mathbf{1 0}$ in good yield.

Further studies of the iron-catalyzed carbomagnesiation may reveal intimate details of the reaction mechanism. In the meantime the method offers an efficient and stereoselective synthesis of tri- and tetrasubstituted olefins. Of note, the carbometalation reported here yields Z-configured allylic and homoallylic olefins. In contrast, the opposite olefin geometry is obtained from procedures based on carbometalation of terminal alkynes followed by trapping with aldehydes or epoxides. ${ }^{1}$

\section{Supplementary Material}

Refer to Web version on PubMed Central for supplementary material.

\section{Acknowledgements}

Financial support from the NIGMS (GM074822) and UT Southwestern (J.M.R. is a Southwestern Medical Foundation Scholar in Biomedical Research).

\section{References}

1. (a) Negishi E. Acc Chem Res 1987;20:65-72. (b) Lipshutz BH, Sengupta S. Org React 1992;41:135631.(c)MarekINormantJDiederichFStangPMetal-Catalyzed Cross-Coupling ReactionsWileyVCHGreat Britain 1998271283 (d) Zhang D, Ready JM. Org Lett 2005;7:5681-5683. [PubMed: 16321021]see also (e) Wipf P, Jahn H. Tetrahedron 1996;52:12853-12910.

2. (a) Fallis AG, Forgione P. Tetrahedron 2001;57:5899-5913. (b) Forgione P, Fallis AG. Tetrahedron Lett 2000;41:11-15. (c) Forgione P, Wilson PD, Fallis AG. Tetrahedron Lett 2000;41:17-20. (d) Ryan J, Micalizio GC. J Am Chem Soc 2006;128:2764-2765. [PubMed: 16506731] (e) Okada K, Oshima 
K, Utimoto K. J Am Chem Soc 1996;118:6076-6077. (f) Eisch JJ, Merkley JH. J Organomet Chem 1969;20:P27-P31. (g) Hojo M, Murakami Y, Aihara H, Sakuragi R, Baba Y, Hosomi A. Angew Chem Int Ed 2001;40:621-623. (h) Ewing JC, Ferguson GS, Moore DW, Schultz FW, Thompson DW. J Org Chem 1985;50:2124-2128.

3. (a) Douboudin JG, Jousseaume B, Bonakdar A, Saux A. J Organomet Chem 1979;168:227-242. (b) Lu Z, Ma S. J Org Chem 2006;71:2655-2660. [PubMed: 16555817]

4. In some cases, dppe was not necessary.

5. We speculate that the $\mathrm{CuBr}$ in Table 2, entry 11 facilitates transmetalation from (vinyl)Fe to (vinyl) $\mathrm{Cu}$. See Shirakawa E, Yamagami T, Kimura T, Yamaguchi S, Hayashi T. J Am Chem Soc 2005;127:17164-17165. [PubMed: 16332046]

6. (a) Snider B, Karras M, Conn RSE. J Am Chem Soc 1978;100:4624-4626. (b) Sato F, Ishikawa H, Watanabe H, Miyake T, Sato M. Chem Commun 1981:718-720. (c) Smith JM, Lachicotte RJ, Holland PL. J Am Chem Soc 2003;125:15752-15753. [PubMed: 14677959]

7. Notes: (a) ${ }^{i} \mathrm{PrMgCl}$ provides the hydrogenated products in moderate yield. (b) No reaction is observed with ${ }^{t} \mathrm{BuMgCl}$. (c) 4 -Octyne does not react with $\mathrm{CH}_{3} \mathrm{MgBr}$ in the presence of $\mathrm{Fe}$ (III) catalysts while cis-hydrogenation is observed with $\mathrm{EtMgBr}$ in the presence of $\mathrm{Fe}(\mathrm{acac})_{3}$. (d) Reactions involving the methyl ether of $1 \mathrm{a}, \mathrm{CH}_{3} \mathrm{MgBr}$ and $\mathrm{Fe}(\mathrm{acac})_{3}$ generate complex mixtures.

8. (a) Treating the alkoxide of 1a with 2 equiv $\mathrm{Fe}\left(\mathrm{CH}_{3}\right)_{3}$ (prepared as in ref $8 \mathrm{~b}$ ) afforded a 3:4 ratio of 2a:3a (100\% conv). Fe(0), as prepared in ref $8 \mathrm{~b}$, did not catalyze the methylation of 1a. (b) Krafft ME, Holton RA. J Org Chem 1984;49:3669-3670.

9. Bogdanovic B, Schwickardi M. Angew Chem Int Ed 2000;39:4610-4612.

10. (a) Aleandri LE, Bogdanovic B, Bons P, Durr C, Gaidies A, Hartwig T, Huckett SC, Lagarden M, Wilczok U, Brand RA. Chem Mater 1995;7:1153-1170. (b) Furstner A, Leitner A, Mendez M, Krause H. J Am Chem Soc 2002;124:13856-13863. [PubMed: 12431116]

$J$ Am Chem Soc. Author manuscript; available in PMC 2008 September 15. 


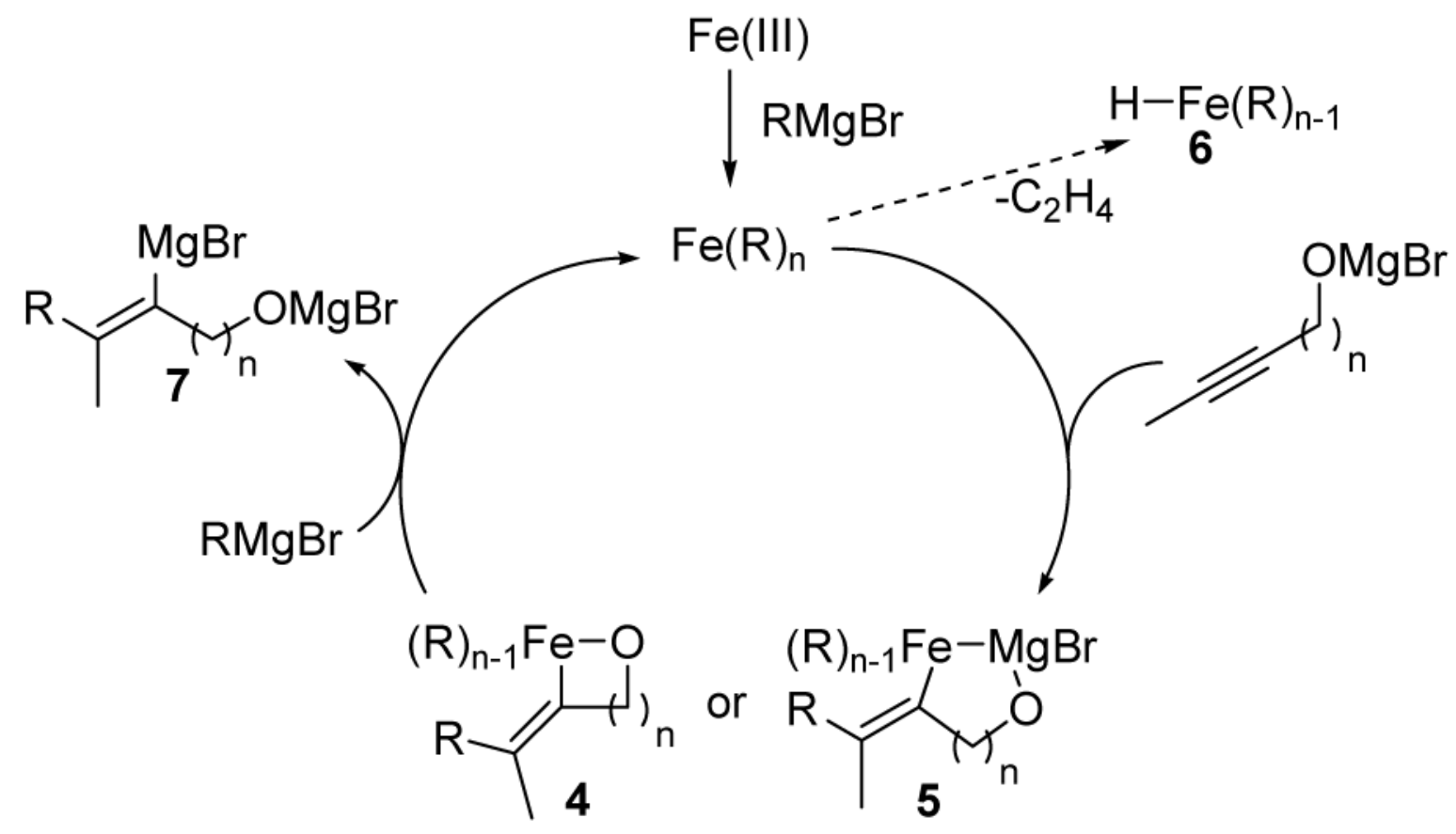

Scheme 1. 

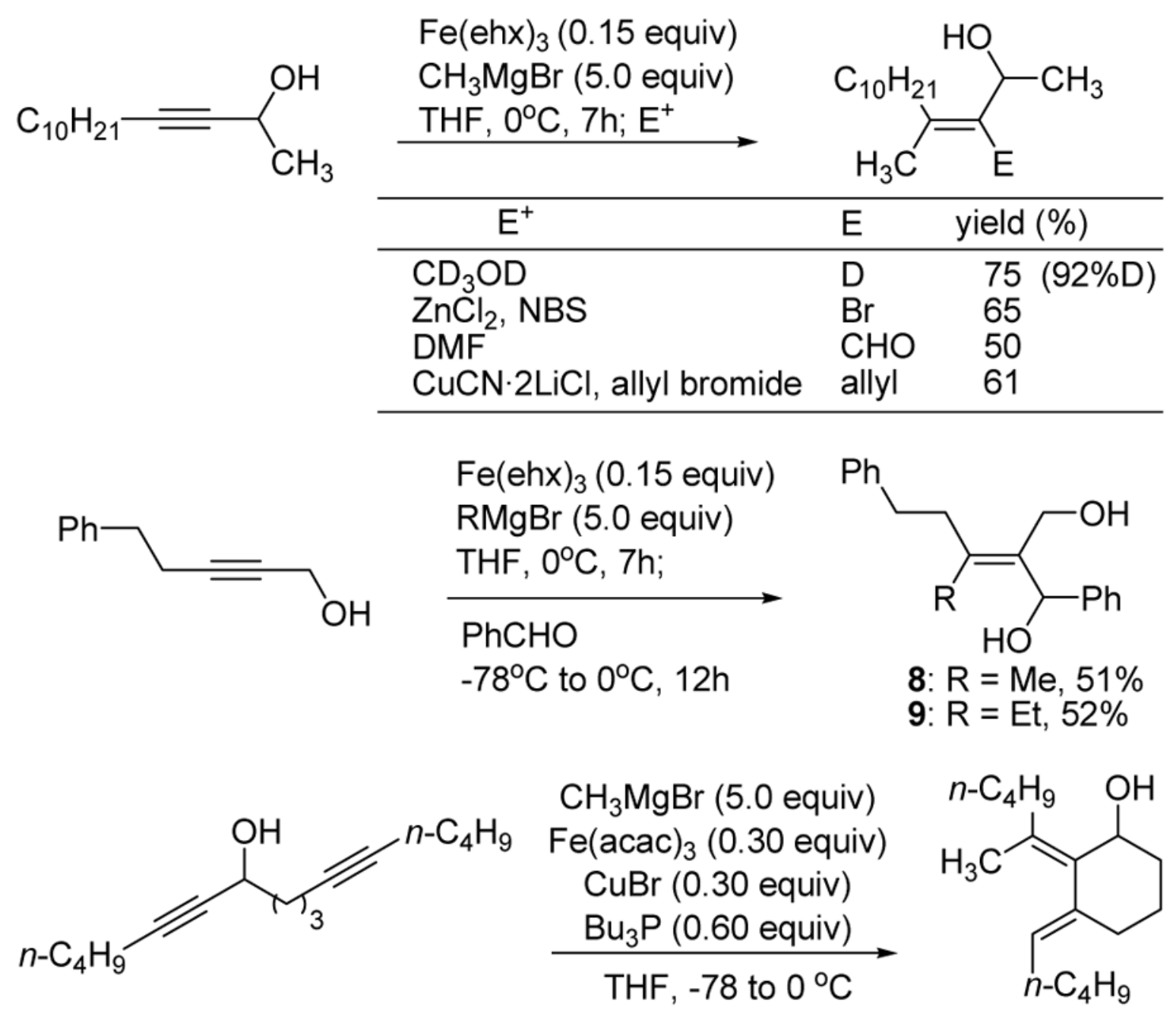

8: $\mathrm{R}=\mathrm{Me}, 51 \%$

9: $\mathrm{R}=\mathrm{Et}, 52 \%$

Synthesis of tetrasubstituted olefins.

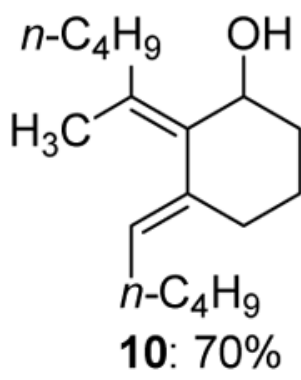

Scheme 2. 


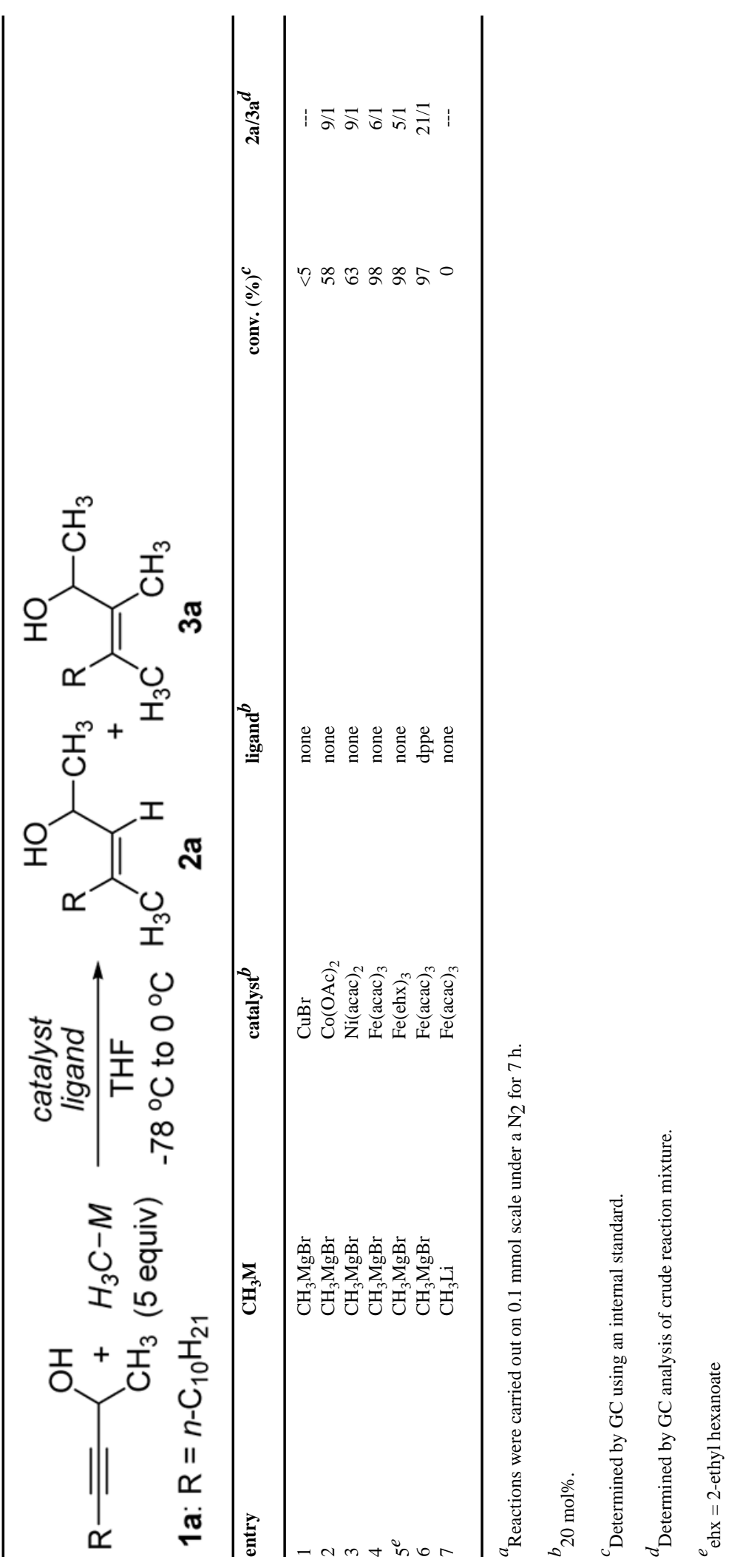


Table 2

Iron-catalyzed carbomagnesiation of propargylic and homopropargylic alcohols. ${ }^{a}$

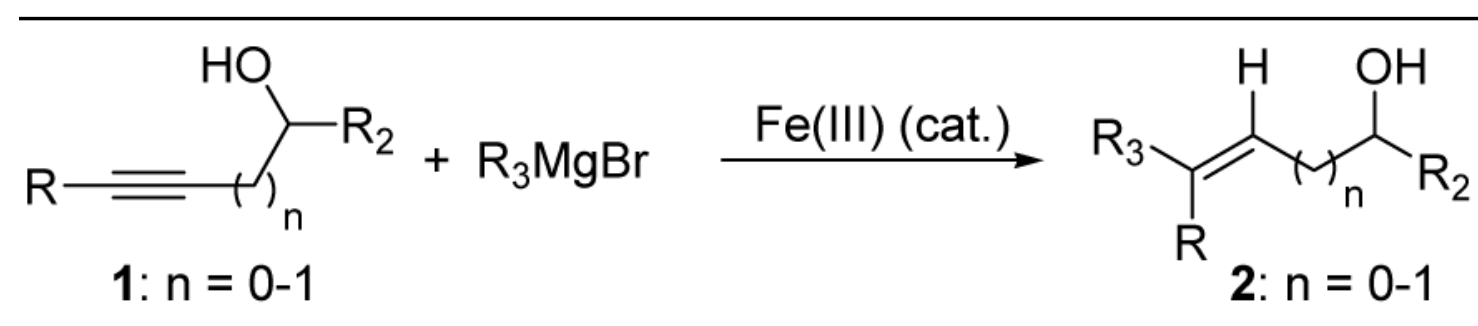

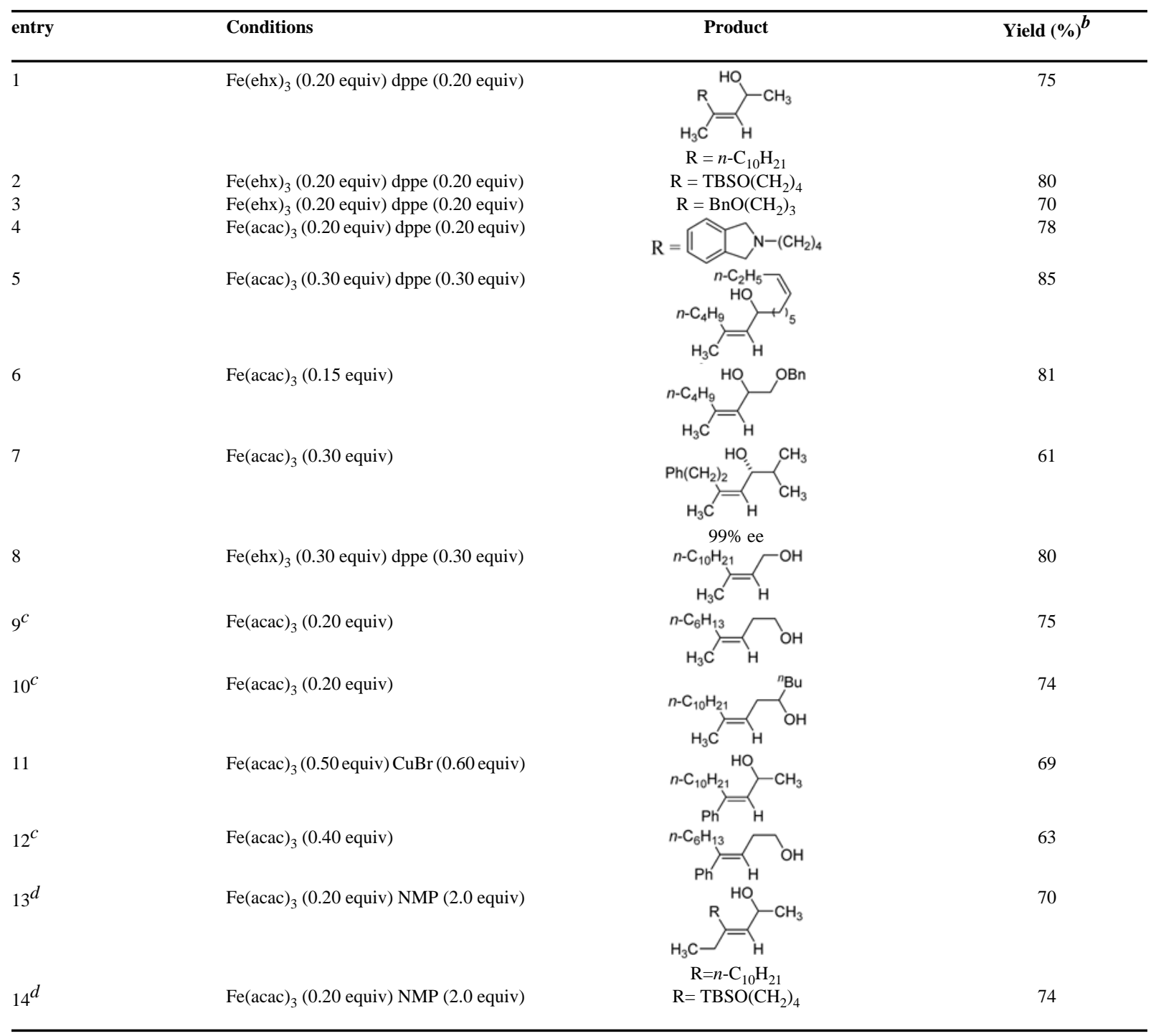

${ }^{a}$ Reactions carried out in THF $\left(0.1 \mathrm{M}\right.$ in substrate) using 5.0 equivalents of $\mathrm{RMgBr}$ at $0{ }^{\circ} \mathrm{C}$ for $7 \mathrm{~h}$ unless otherwise indicated.

$b_{\text {Isolated yield. }}$

${ }^{c}$ In toluene at $23^{\circ} \mathrm{C}$.

$d_{\mathrm{NMP}}=N$-methyl pyrrolidine. 